\title{
Examination for cardiac malformations at six weeks of age
}

\author{
Jill Gregory, Alison Emslie, Jonathan Wyllie, Christopher Wren
}

\begin{abstract}
Aim-To attempt to define the prevalence and significance of murmurs detected on routine clinical examination at six to eight weeks.

Methods-A retrospective review of the results of routine clinical examination of a cohort of 6 to 8 week old babies resident in Newcastle upon Tyne, was carried out in two 12 month periods. All cardiac defects diagnosed in infancy in the same cohort were ascertained.

Results-7132 babies were eligible for routine examination; $83 \%$ of these were examined. Murmurs were heard in 47 of 5395 babies and in 11 of 25 referred for evaluation congenital heart disease was found. The six to eight week examination led to diagnosis of 11 of 35 cases $(31 \%)$ of congenital heart disease in the study population.

Conclusions-Nearly one baby in 100 had a murmur on routine examination at six to eight weeks. Nearly half of those with murmurs who were referred had a structural cardiovascular malformation. (Arch Dis Child Fetal Neonatal Ed 1999;80:F46-F48)
\end{abstract}

Keywords: congenital heart disease; screening; cardiac murmur
Examination for signs of cardiovascular malformation at six to eight weeks is part of the routine health surveillance of young infants. ${ }^{1}$ Although the presence of a murmur at this age is potentially clinically significant and "prompt referral" for specialist assessment is recommended in national guidelines, ${ }^{1}$ the efficacy of such a policy has not been formally evaluated. This study examines the performance of routine clinical examination at six to eight weeks for the detection of cardiovascular malformations.

\section{Methods}

The study was originally designed in two parts: a retrospective review of babies examined between January and December 1991, and a prospective assessment of those examined between July 1993 and June 1994. After analysis of the first cohort, general practitioners and community paediatricians were advised to refer all babies with murmurs in the second 12 month period for specialist examination. However, this advice was not followed and there was no difference in referral practice between the two groups, so the results are presented together.

All live born babies whose mothers were resident in Newcastle upon Tyne and who were eligible for examination at 6 to 8 weeks of age

\section{Department of Community Paediatrics Newcastle City Health NHS Trust J Gregory}

Department of Clinical Audit

A Emslie

Department of

Paediatrics

South Cleveland

Hospital,

Middlesbrough

J Wyllie

Department of Paediatric Cardiology Freeman Hospital, Newcastle upon Tyne

C Wren

Correspondence to: Dr Christopher Wren Department of Paediatric Cardiology

Freeman Hospital

Newcastle upon Tyne NE7 7DN.

Accepted 26 June 1998

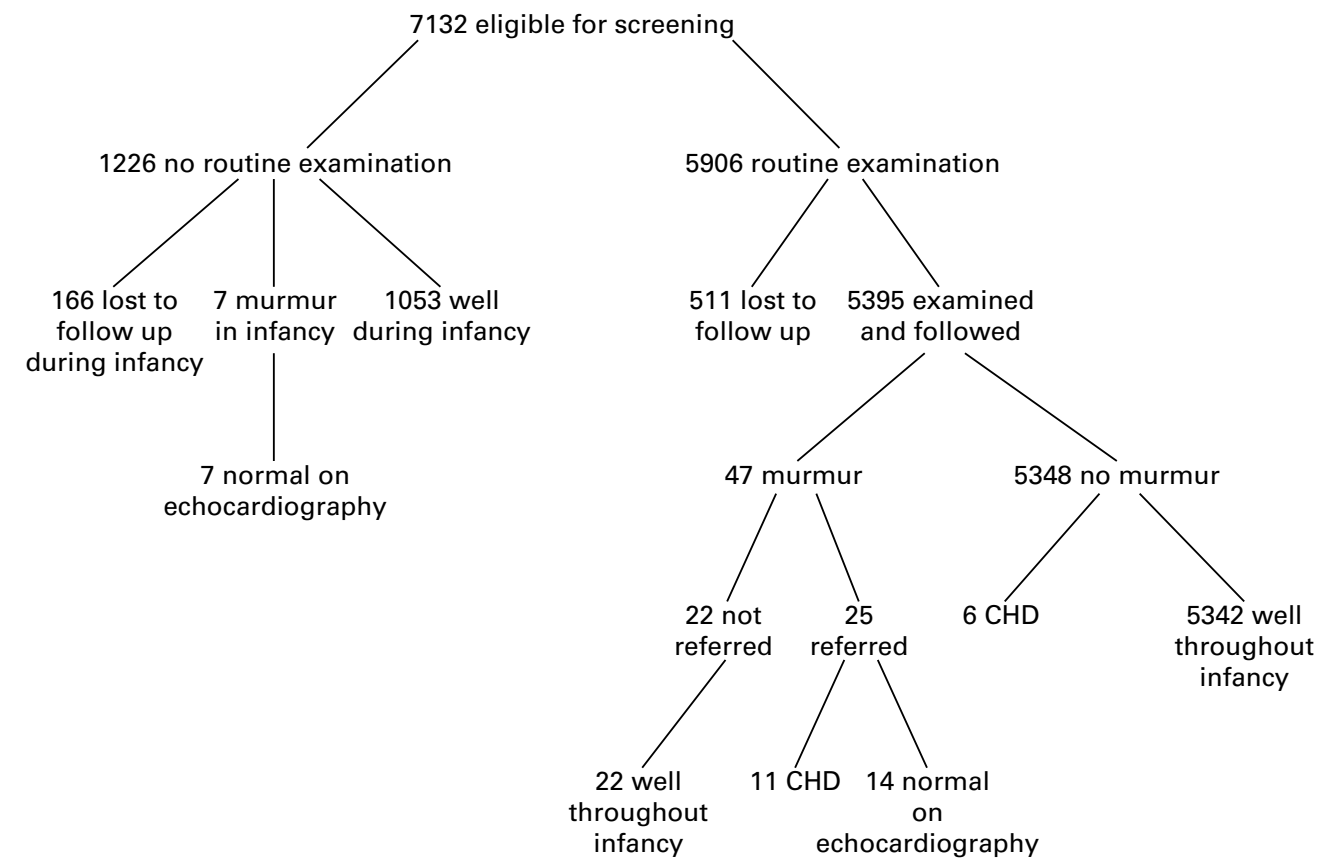

Figure 1 Flow diagram showing progress of the study from six to eight weeks to 12 months of age; $C H D=$ coronary heart disease. 
Table 1 Numbers and types of cardiovascular malformations identified in study population

\begin{tabular}{lll}
\hline $\begin{array}{l}\text { Diagnosis } \\
\text { Before } 6 \text { weeks }\end{array}$ & VSD & 12 \\
& PDA & 2 \\
& AVSD & 1 \\
COA & 1 \\
PS & 1 \\
PA/VSD & 1 \\
6 to 8 weeks & VSD & 7 \\
& AVSD & 2 \\
TGA & 1 \\
After 6 to 8 weeks & TOF & 1 \\
& PS & 3 \\
& VSD & 2 \\
& COA+VS D & 1
\end{tabular}

VSD = ventricular septal defect; PDA = patent ductus arteriosus; AVSD = atrioventricular septal defect; $\mathrm{COA}=$ coarctation of aorta; PS = pulmonary valve stenosis; PA/VSD = pulmonary atresia with ventricular septal defect; TGA = transposition of the great arteries; TOF = tetralogy of Fallot.

between 1 January 1991 and 31 December 1991 and between 1 July 1993 and 30 June 1994 were identified. Routine clinical examination was performed by general practitioners and community paediatricians. The results of the examination (when performed) were identified by retrospective examination of records held at the Community Records Department, and the outcome of all those with murmurs was ascertained from the paediatric cardiology database.

The regional paediatric cardiology database provided information on all infants in the same cohort with congenital heart disease diagnosed by the age of 12 months. The database incorporates information from the regional perinatal and infant mortality survey to identify all cases dying before diagnosis. ${ }^{23}$ The Freeman Hospital information system provided details of all infants evaluated for murmurs which were found to be benign. Congenital heart disease was defined as "a gross structural abnormality of the heart or intrathoracic vessels that is actually or potentially of functional significance". ${ }^{4}$

\section{Results}

In the two years of the study 7132 babies were eligible for routine clinical examination at six to eight weeks (fig 1). Of these 5906, (83\%) were screened but 511 were lost to follow up or moved away before 12 months of age. The results of examination of the remaining 5395 are shown in the fig 1 . Murmurs were heard in 47 babies $(0.9 \%)$, but only 25 were referred for paediatric cardiological assessment. There is no information on how these babies were selected. As fig 1 shows, 11 of the 25 babies with murmurs who were referred had congenital heart disease and six with no murmur were subsequently found to have a cardiac defect during infancy. All diagnoses were confirmed by echocardiography. The 22 who were not referred remained well throughout infancy, and, as far as can be ascertained from the records, the murmurs all resolved spontaneously.

Of 1226 babies (17\%) who did not undergo routine examination, seven $(0.7 \%)$ were subsequently referred for evaluation of a murmur,

\section{Key messages}

- Only $83 \%$ of eligible babies received routine examination for signs of heart disease and only $56 \%$ between 6 and 8 weeks of age.

- Murmurs were heard in roughly one baby in $100(0.9 \%)$.

- About one quarter of babies with murmurs had structural heart disease but only about half were referred.

- Referral of all babies with murmurs at this age for expert evaluation is recommended.

1053 were normal throughout infancy (in that they remained resident in Newcastle and did not present with heart disease during this time) and 166 were lost to follow up or moved away (fig 1).

Routine examination was not always carried out between 6 and 8 weeks of age. In this study $131(2 \%)$ underwent what was considered to be a routine examination before 6 weeks, 1171 $(20 \%)$ between 8 and 10 weeks, and $607(10 \%)$ beyond 10 weeks.

Because of the study design it is not possible to define a birth cohort. However, congenital heart disease was recognised in babies born and resident in the catchment area throughout infancy in $18(51 \%)$ before 6 weeks of age, as a result of the six week examination in $11(31 \%)$, and between six weeks and 12 months of age in six $(17 \%)$. Specific diagnoses made at various stages are shown in table 1.

\section{Discussion}

Routine examination for signs of congenital heart disease at 6 to 8 weeks of age is recommended in national guidelines ${ }^{1}$ and is aimed at improving the outcome for babies with cardiovascular malformations, by detection at a pre-symptomatic stage. Although this policy potentially meets most of the criteria for a screening test, ${ }^{5}$ its efficacy has not been formally evaluated before. Many babies with clinically significant abnormalities will have been identified before 6 weeks of age, although others may remain asymptomatic. ${ }^{6}$ In a retrospective study of the presentation of a cohort of babies with cardiovascular malformations diagnosed in infancy, only $35 \%$ of those sent home without a definite diagnosis had achieved a diagnosis by 6 weeks of age. ${ }^{6}$ After neonatal examination the six to eight week examination provides the only opportunity for detection of heart disease in infancy, other than opportunistic recognition of a murmur or the development of symptoms.

In retrospect, the design of our study could have been improved, particularly by the identification of a birth cohort which could then be followed up through infancy. Only $83 \%$ of babies who were eligible for examination underwent screening at all, and only $56 \%$ were examined between 6 and 8 weeks of age. Another weakness is that only $76 \%$ of the babies in the study population were examined at 6 to 8 weeks of age and were then available 
for follow up throughout infancy (fig 1). Only $53 \%$ of those with murmurs were referred for expert assessment.

The 22 infants with murmurs who were not referred, the 5348 examined and found to have no murmur, and the 1053 not screened but who remained resident in the area can be assumed to have had no major cardiovascular problem as they were not referred to the only paediatric cardiology unit in the region and did not die. Whether they might have had a minor abnormality or none at all remains unknown. Most clinically significant congenital heart disease presents in infancy, ${ }^{78}$ although some malformations, especially atrial septal defects and persistent arterial ducts, may not present until later. However, this study was aimed only at identifying problems which presented in infancy.

In this study $31 \%$ of all diagnoses made in infancy resulted from the six to eight week examination and $44 \%$ of babies with a murmur at this age turned out to have congenital heart disease. Routine examination did identify some clinically significant cardiac malformations but other significant problems were not detected (table 1). As with the neonatal examination, ${ }^{69}$ it is important to recognise that absence of abnormal signs does not exclude abnormality.

Because of the design limitations we cannot clearly establish the population prevalence of murmurs at this age, although there is no obvious reason why the babies examined and followed up should be different from those who were not. For similar reasons it is difficult to define a precise birth prevalence of congenital heart disease diagnosed in infancy in this study. However, the figure of 35 cases from around 7000 births is consistent with those of previous studies with confirmed diagnoses only and ascertainment limited to infancy. ${ }^{6}{ }^{10-12}$ Despite its limitations, the study provides useful information for assessment of the routine six to eight week examination and for guidelines on what should be done if a murmur is heard.

This study set out to examine the efficacy of routine examination as it is performed rather than as it ideally should be performed. It highlights major deficiencies in the six to eight week examination as it is currently practised. Screening is performed with poor record keeping, inconsistent action in the face of an abnormality, and no assessment of outcome. There is obviously room for improvement.

The paediatric cardiology database is supported by the Children's Heart Unit Fund.

1 Hall DMB, ed. Health for all children. Report of the third joint working party on child health surveillance. Oxford: joint working party on child hea

2 Northern Regional Survey Screening Group. Fetal abnormality, an audit of its recognition and management. Arch Dis Child 1992;67:F770-4

3 Northern Regional Health Authority Coordinating Group. Perinatal mortality: a continuing collaborative regional group. BMF 1984;288: 1717-20.

4 Mitchell SC, Korones SB, Berendes HW. Congenital heart disease in 56,109 births. Circulation 1971:43:323-32.

5 Hall DMB, Michel JM. Screening in infancy. Arch Dis Child 1995;72:93-6.

6 Wren C, Richmond S, Donaldson L. Presentation of congenital heart disease in infancy: implications for congenital heart disease in infancy: implications
routine examination. Arch Dis Child 1999;80:F49-53.

7 Samanek M, Zdenek S, Bozena Z, Hrobonova V, Voriskova M, Skovranec J. Prevalence, treatment and outcome of heart disease in live-born children: a prospective analysis of 91,823 live-born children. Pediatr Cardiol 1989;10:20511.

8 Hoffman JIE. Incidence of congenital heart disease: I. Postnatal incidence. Pediatr Cardiol 1995;16:103-13.

9 Silove ED. Assessment and management of congenital heart disease in the newborn by the district paediatrician. Arch Dis Child 1994;70:F71-4.

10 Ferencz C, Rubin JD, McCarter RJ, et al. Congenital heart disease: prevalence at live birth. $A m \mathcal{J}$ Epidemiol 1985;121:31-6.

11 Grabitz RG, Joffres MR, Collins-Nakai RL. Congenital heart disease: incidence in the first year of life. Am $\mathcal{F}$ Epidemiol 1988;128:381-8.

12 Kidd SA, Lancaster PAL, McCredie RM. The incidence of congenital heart defects in the first year of life. $\mathcal{F}$ Paediatr Child Health 1993;29:344-9. 